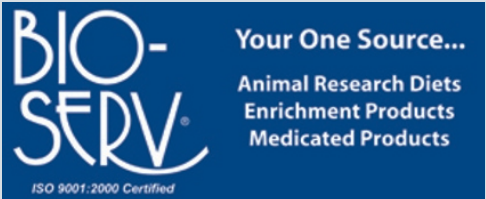

\title{
Bio-Serv's 'special needs' diets provide solutions to special challenges in research
}

Yisheng Bai, PhD \& Karen M. Froberg-Fejko, VMD

Nutritionally balanced standard diets are suitable for many lab animals at various developmental stages. But certain animal and protocol conditions-including genetic alteration; breeding difficulty; digestive upset; post-operative intervention and dehydration; and diabetes and obesity-create a requirement for 'special needs' products to ensure animal survival and encourage reliable research outcomes. With input from technicians and scientists from numerous research facilities, Bio-Serv has worked diligently to create a line of diets, enrichment treats and supplements for research animals with special needs. Here we provide an overview of what we have learned about some of these conditions and the recommended solutions we have developed to address them.

\section{Genetic alteration}

Transgenic and knock-out mice may have phenotypes that limit their ability to eat and drink normally. Genetic changes may result in absence of teeth, malocclusion or neurotic behavior, which affect food and water intake. Such animals require a soft, palatable diet to ensure proper nutrition. Transgenic Dough Diet ${ }^{\mathrm{TM}}$ is a sterile, highly palatable, bacon-flavored, nutritionally condensed diet. Nutra-Gel ${ }^{\mathrm{TM}}$ is a sterile, nutritionally complete diet that supplies both food and water, eliminating the need for messy mush diets. Both diets can be offered at the cage level to provide easy access for the animals and to help stimulate their appetites. These diets should be changed daily to maintain freshness and prevent drying, especially when using ventilated caging.

\section{Breeding}

Breeding challenges create a host of special needs, especially for some transgenic and knock-out rodent lines. Love Mash ${ }^{\mathrm{TM}}$ was developed many years ago by a large rodent breeder and has been used successfully to enhance reproductive performance in males and females. Empirical data suggest that Love Mash increases litter size and pup survival rates. Its success relies on the fat composition of the diet. Love Mash is offered in the form of sterile, soft pellets with a diameter of $0.5 \mathrm{in}$. The pellets can be given to rodents ad libitum or as a supplement, starting 2 weeks before mating and continuing through weaning of the pups.

Stress is a serious problem in many dams, and decreasing stress by offering a sheltering device such as the Mouse $\operatorname{Igloo}^{\oplus}$ (Fig. 1) is essential. The Mouse Igloo is available with or without a floor. The floor allows the mother and pups to be transported to a new

Bio-Serv, One 8th St., Ste. 1, Frenchtown, NJ 08825. Correspondence should be addressed to K.M.F.-F. (kfroberg@bio-serv.com).

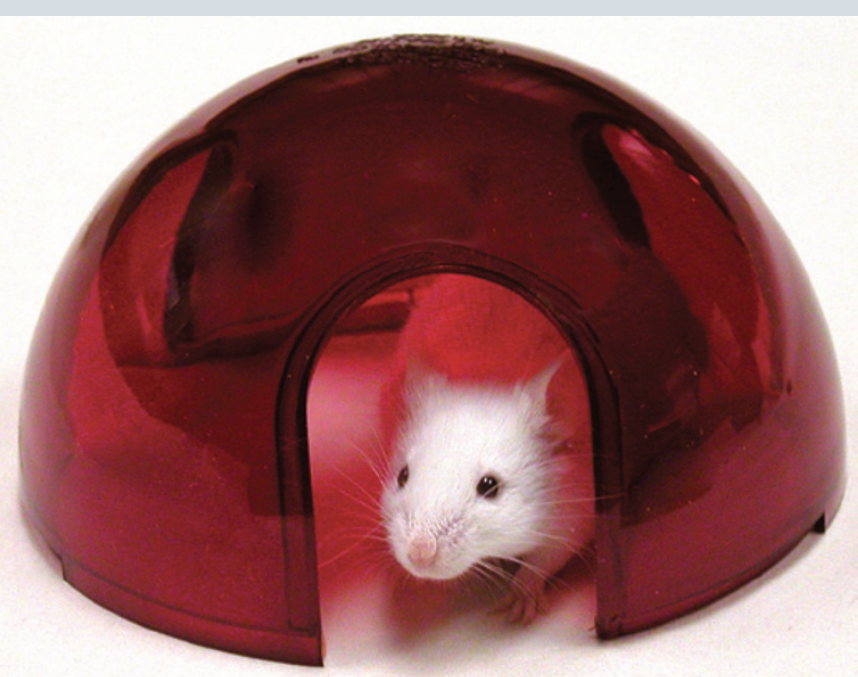

FIGURE 1 | Offering a Mouse Igloo ${ }^{\circledR}$ can reduce stress in breeding females. Use with the optional floor for stress-free transport of mother and pups during cage changes.

cage more easily. As an added benefit, the Mouse Igloo decreases physiological stress in pups by directing airflow away from them in ventilated cages.

Cannibalism is another problem commonly encountered in nervous dams. A great way to prevent cannibalism is to offer Supreme Mini Treats ${ }^{\mathrm{TM}}$ (Chocolate or Very Berry flavors) to a dam just after she delivers her pups. She will readily eat the treats, sparing her pups.

Neonatal care poses its own unique challenges. Appropriate milk replacement products are essential for proper growth and 


\section{ADVERTISING FEATURE}

development of newborns. Bio-Serv offers milk replacements for both non-human primates and rodents. Primilac ${ }^{\mathrm{TM}}$ (Fig. 2) is a milk substitute designed to meet the high metabolic rate and increased nutrient requirements of growing New and Old World infant non-human primates. Compared with human formulas, Primilac contains more nutrients, such as selenium, chromium and fluoride, and a greater amount of vitamin C. Rodent Milk Substitute $^{\mathrm{TM}}$ is useful for transgenic or knock-out neonates whose dams fail to produce enough milk during lactation. This milk substitute uses hydrolyzed casein as its protein source for increased digestibility. Both Primilac and Rodent Milk Substitute come in dry powder form and are easy to prepare by simply adding water. In addition, for rodent pups, offering Bacon Softies ${ }^{\mathrm{TM}}$ at the cage level as a transitioning diet can help to boost their strength and weight gain.

\section{Digestive upset}

Diarrhea is a common digestive ailment among non-human primates resulting from food allergies, bowel disorders, infectious diseases, congenital anomalies and stress. Sensitive System $\operatorname{Diet}^{\mathrm{TM}}$ (Fig. 3) is made with a limited number of highly digestible ingredients not typically used in standard monkey chows, which makes it an excellent choice for non-human primates suffering with gastrointestinal upsets, regardless of the underlying etiology.

\section{Post-operative intervention}

Post-operative care and dehydration require special intervention. Bio-Serv has developed several products to help animals through the critical post-operative phase and bring them back to health. Soft diets, including Bacon Softies for rodents and PRIMA-Burgers $^{\mathrm{TM}}$, Monkey Dough ${ }^{\mathrm{TM}}$ and Banana, Cherry and

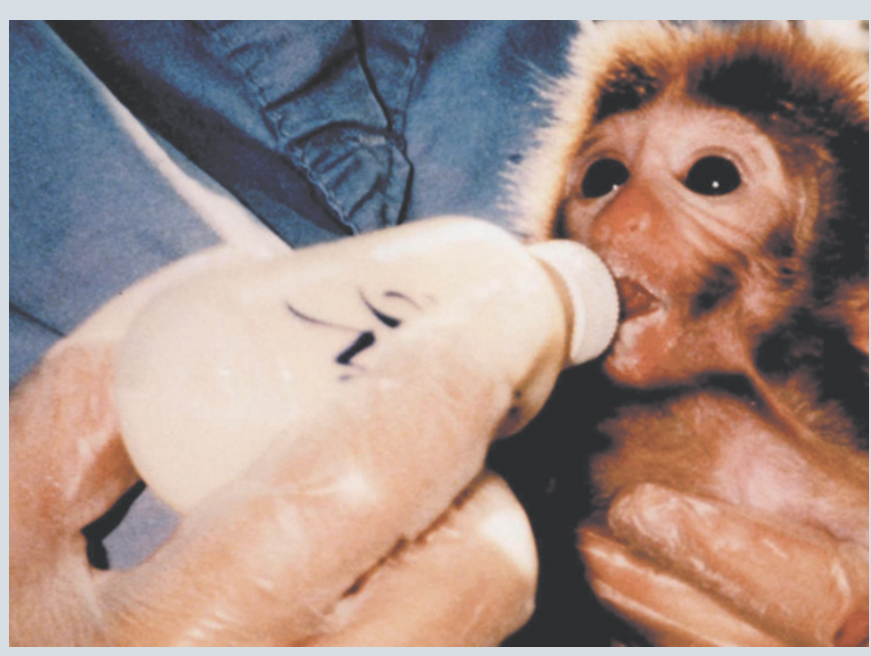

FIGURE 2 | Primilac ${ }^{\text {T"M }}$ is a milk substitute specially developed for the metabolism and nutritional needs of infant New and Old World primates.

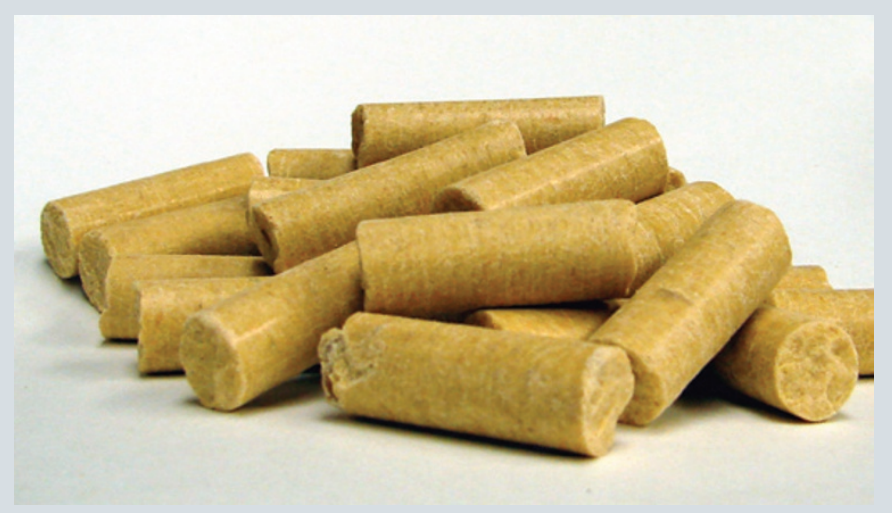

FIGURE 3 | The Sensitive System Diet ${ }^{\text {'m }}$, made with easily digestible ingredients not typically found in monkey chow, is made for primates suffering from digestive upset.

Marshmallow Softies for non-human primates, are excellent nutritional options that stimulate appetite in anorexic animals. These highly palatable and nutritious diets replenish essential nutrients. PRANG ${ }^{\mathrm{TM}}$ is an electrolyte replacement drink to be used for non-human primates and rabbits that need critical rehydration therapy. PRANG is available in six fruit flavors and contains three times the electrolytes found in Gatorade. To ensure freshness, PRANG is sold in powder form and mixed with water before being offered to animals.

\section{Diabetes and obesity}

Diabetic animal models and overweight animals can benefit from our line of Sugar Free (Calorie Free) products. Sugar Free Prang, like regular PRANG, is an electrolyte replacement drink that works well for stressed or debilitated non-human primates and rabbits. Sugar Free (Calorie Free) Wafers are 3-g, banana-flavored treats that conveniently fit into many of Bio-Serv's enrichment devices. Sugar Free (Calorie Free) Treats are 650-mg, piña colada-flavored treats for multiple species including non-human primates, pigs, rabbits, guinea pigs, rats and mice. For non-diabetic overweight species, Skinny Mini's ${ }^{\mathrm{TM}}$ are fortified, low-calorie, 1-g, cherry-flavored treats.

\section{Company profile}

Bio-Serv helps customers provide their research animals with the highest level of care by making available a variety of innovative products for all laboratory animal species. With more than 36 years of experience and a well-trained, professional staff, Bio-Serv can provide solutions for animals with special needs.

This article was submitted to Lab Animal by a commercial organization and has not been peer-reviewed. Lab Animal takes no responsibility for the accuracy or relevancy of the information provided therein. 